\title{
吲哚苄位碳正离子引发的串联环化反应的普适性和机理探究
}

\author{
高中润†王 媛†宋 航徐正仁* 贾彦兴* \\ (北京大学药学院 天然药物及仿生药物国家重点实验室 北京 100191)
}

\begin{abstract}
摘要 对在 $\alpha$-环匹阿尼酸 $(\alpha-\mathrm{CPA})$ 和 speradine $\mathrm{C}$ 全合成中发展的仿生吲哚苄位碳正离子引发的串联环化反应的普适性 和机理进行了研究, 实验结果表明, 该芐位碳正离子串联环化反应在含有吲槑的底物中能顺利发生, 说明吲哚氮原子 的共轭效应在吲哚苄位碳正阳离子串联环化反应中起到了关键性的作用.
\end{abstract}

关键词 反应机理; 串联环化; 芐位碳正离子; 吲梠; 共轭效应

\section{Cascade Cyclization Reaction Initiated by Benzylic Carbocation of Indole: Scope and Mechanistic Insights}

\author{
Gao, Zhongrun ${ }^{\dagger} \quad$ Wang, Yuan ${ }^{\dagger} \quad$ Song, Hang $\quad \mathrm{Xu}$, Zhengren* Jia, Yanxing* \\ (State Key Laboratory of Natural and Biomimetic Drugs, School of Pharmaceutical Sciences, \\ Peking University, Beijing 100191)
}

\begin{abstract}
During the course of the total synthesis of $\alpha$-cyclopianic acid ( $\alpha$-CPA) and speradine C, our research group have developed a biomimetic cascade cyclization reaction initiated by the benzylic carbocation of indole. In this paper, the scope and the reaction mechanism of the cascade cyclization reaction were studied. The experimental results show that the cascade cyclization reaction occurrs at the benzylic carbocation of indole, indicating that the conjugation effect of the indole nitrogen atom plays a key role in the cascade cyclization of benzylic carbocations.
\end{abstract}

Keywords mechanism; cascade cyclization; benzylic carbocation; indole; conjugation effect

仿生合成可以简化合成步骤，提高合成效率. 近些 年, 我们课题组利用仿生合成策略，实现了一系列复杂 二萜和吲哚生物碱的高效全合成 ${ }^{[1-9]}$. 作为正在进行的 简明高效合成 3,4-桥环吲哚类生物碱研究的一部

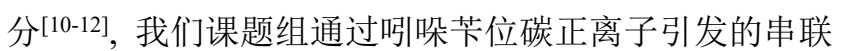
环化一步构建 $\mathrm{C} / \mathrm{D}$ 环作为关键仿生步骤，仅用 7 步和 10 步就分别实现了 $\alpha$-环匹阿尼酸 $(\alpha$-cyclopiazonic acid, $\alpha$-CPA，1)及其相关高氧化态天然产物 speradine C (2)的 简洁高效全合成 ${ }^{[7,9]}$. 由于类似的由芐位碳正离子引发 的仿生串联环化反应未见文献报道, 引起了我们的关注 和兴趣, 决定对其适用范围和机理进行探究.

如 Scheme 1a 所示, 生物合成途径研究表明 ${ }^{[13]}, \beta$ 环匹阿尼酸 $(\beta$-cyclopiazonic acid, $\beta$-CPA, 3) 是 $\alpha$-CPA 的 直接生物合成前体. $\beta$-CPA 在黄素依赖性单胺氧化酶 MaoA (CpaO) 作用下产生吲哚芐位碳正离子中间体 $\mathbf{4}$,
其随后发生碳正离子引发的级联环化一步构建 $\mathrm{C} / \mathrm{D}$ 环 生成 $\alpha$-CPA.

如 Scheme $1 b$ 所示, $\mathrm{Liu}^{[7]}$ 和 Shi 等 ${ }^{[9]}$ 在实验过程中发 现，化合物 5 在催化量的 $\mathrm{Tf}_{2} \mathrm{NH}$ 作用下, $5 \mathrm{~min}$ 就完全消 失, 生成了单一构型的 $E$ 式脱氢色氨酸衍生物 $\mathbf{6}$ 和微量 的环化产物 7; 随着时间延长, 化合物 6 逐渐消失, 环化 产物 7 逐渐增加. 此外, 对照实验研究表明, 化合物 6 在该条件下也能发生环化得到化合物 7.

我们对该反应的过程进行了推测, 如 Scheme 2 所 示. 该反应过程可能有吲哚氮)原子不参与(path A, 普通 芐位碳正离子)和吲哚氮原子参与(path $\mathrm{B}$, 更稳定苄位 碳正离子)两种途径. 在 path $\mathrm{A}$ 途径中, 质子酸直接活化 化合物 6 的羰基，诱导烯烃对不饱和内酯加成生成碳正 离子中间体 Int-1，并被氮原子捕获得到化合物 7; 类似 的烯烃对不饱和酮和不饱和双酯的加成已有报道 ${ }^{[14-17]}$,

\footnotetext{
* Corresponding authors. E-mail: yxjia@bjmu.edu.cn; zhengrenxu@bjmu.edu.cn Received February 1, 2021; revised May 2, 2021; published online May 23, 2021. Project supported by the National Natural Science Foundation of China (No. 21871013). 国家自然科学基金(No. 21871013)资助项目.

$\dagger$ 共同第一作者(These authors contributed equally to this work).
} 
(a)<smiles></smiles><smiles>CCCCC[C@H]1C(=C(C)C)Cc2cccc3[nH]cc1c23</smiles>

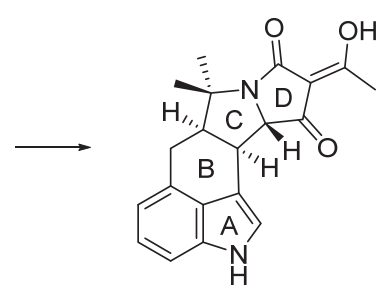

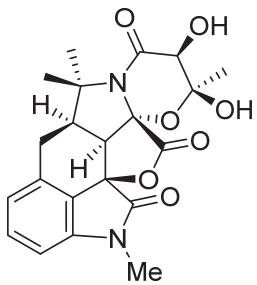

speradine C (2)

(b)<smiles>[R]n1cc(C(O)C(N)C(C)=O)c2c(CC=C(C)C)cccc21</smiles>

5: $R=T s, M e$<smiles>[R]n1cc(C=C(N)[NH2+])c2c(CC=C(C)C)cccc21</smiles>

6: $\mathrm{R}=\mathrm{Ts}, \mathrm{Me}$

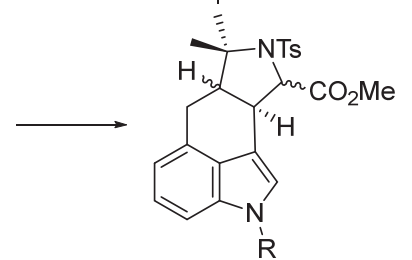

7: $\mathrm{R}=\mathrm{Ts}, \mathrm{Me}$

图式 $1 \alpha$-CPA 的生源合成

Scheme 1 Biosynthesis of $\alpha$-CPA

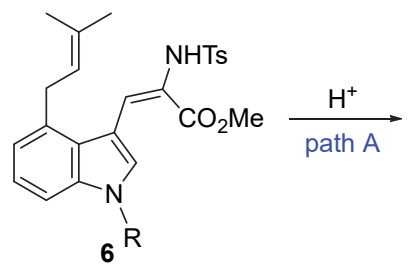

path $\mathrm{B} \mid \mathrm{H}^{+}$<smiles>[R]n1cc(/C=C(/[NH2+][18F])C(=O)OC)c2c(CC=C(C)C)cccc21</smiles><smiles>[R]n1cc(/C(C(=O)[O-])=C(\N)C(=O)OC)c2c(CC=C(C)C)cccc21</smiles>

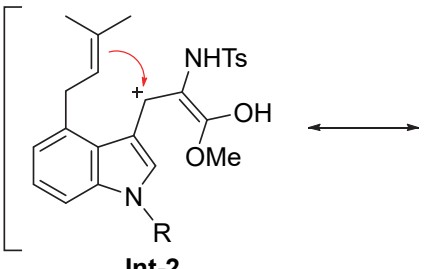<smiles>[R]n1cc2c3c(cccc31)C[C@H](C(C)(C)C)[C@H]2/C(N)=C(/O)OC</smiles>

Int-1

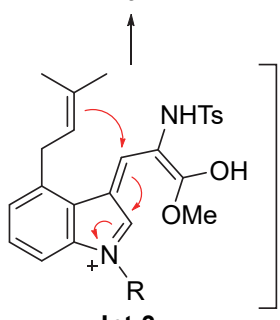<smiles>[R]n1cc2c3c(cccc31)C[C@@H]1[C@H](C)[NH2+][C@@H](C)/C(=C(\O)OC)[C@H]21</smiles>

8

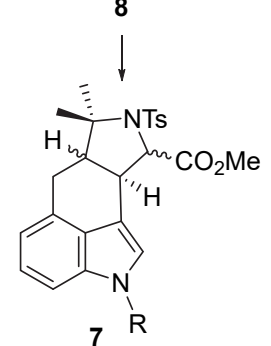

图式 $2[3+2]$ 环加成反应机理推测

Scheme 2 A possible mechanism for [3+2] annulation

而对不饱和单酯的还未见报道. 在 path B 途径中, 化合 物 6 在强酸条件下形成茮位碳正离子 Int-2, 同时, 由于 吲哚氮原子的共轭效应参与稳定苠位碳正离子, 形成含 有吲哚骨架的亚胺阳离子 Int-3, 并引发非官能团化烯 烃的分子内亲核进攻形成碳正离子中间体 Int-1, 后续 发生进一步的环化反应得到化合物 7.

我们认为, 如果该串联反应在构筑并环结构中具有 很好的普适性，不仅可以利用该方法制备系列天然产物 的类似物及简化物, 方便开展进一步的构效关系研究, 而且可以应用于更多天然产物的全合成中.

\section{1 结果与讨论}

上述两种推测的机理中, 如果是按照 path A 的途 径, 吲哚氮原子不参与反应过程, 那么该串联环反应可
能具有较好的普适性, 对于一般的苯环底物甚至是简单 结构底物或可适用; 如果是按照 path B 的途径, 说明吲 哚氮原子的共轭效应参与其中，该反应需要在吲哚类等 特殊骨架中才能进行.

首先设计合成了苯环骨架化合物 10, 如 Scheme 3 所示. 已知化合物 9 $^{[18-19]}$ 经过一步 aldol 反应 ${ }^{[7,9]}$ 顺利得 到环化前体 $\mathbf{1 0}$, 但是化合物 $\mathbf{1 0}$ 在催化量 $\mathrm{Tf}_{2} \mathrm{NH}$ 的存在 下没有得到预期的 $6 / 5 / 5$ 并环环化产物 12 , 而是得到了 氧杂七元环化合物 10 . 结果说明, 化合物 10 在酸性条 件下双键的质子化优先于羟基的消除，苄位碳正离子未 能生成.

于是设计了类似于化合物 6 的不饱和羰基化合物 14, 尝试比较从双键出发是否更容易形成所需的苄位碳 正离子, 如 Scheme 4 所示. 从已知化合物 $13^{[19-20]}$ 出发, 

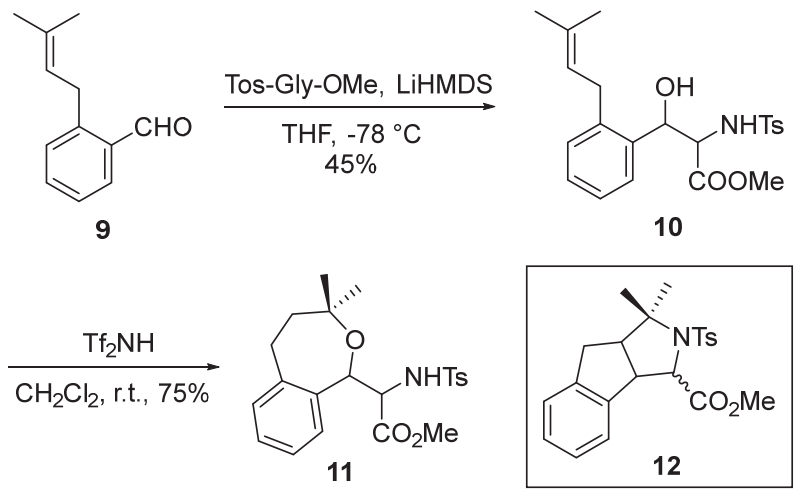

图式 3 化合物 10 的环加成反应尝试

Scheme 3 Attempt on cyclization of compound $\mathbf{1 0}$

经过一步 Wittig 反应 ${ }^{[21-22]}$ 即可得到目标化合物 14. 但 是, 化合物 14 在 $\mathrm{Tf}_{2} \mathrm{NH}$ 的催化下也没有得到预期的环 化产物 16, 而是得到了双键发生傅克反应的产物 15. 结 果说明在化合物 14 的结构中, 从双键出发也难以优先 生成所需的苄位碳正离子.

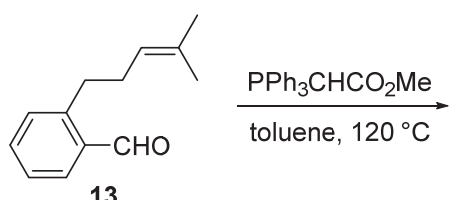

13
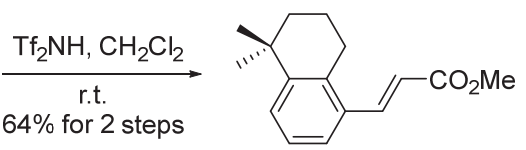

15

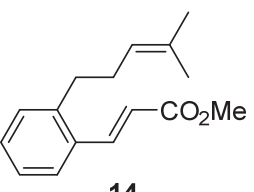

14

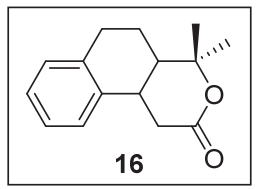

图式 4 化合物 14 的环加成反应尝试

Scheme 4 Attempt on cyclization of compound 14

鉴于芳基对位甲氧基的共轭效应可以稳定苠位碳 正离子, 如 Scheme 5 所示, 从已知化合物 17 ${ }^{[19-20]}$ 出发, 经过一步 Wittig 反应 ${ }^{[22]}$ 得到目标化合物 18. 同化合物 14 的结果一样, 化合物 18 在 $\mathrm{Tf}_{2} \mathrm{NH}$ 的催化下没有得到
预期的环化产物 $\mathbf{2 0}$, 得到的依然是双键发生傅克反应 的产物 19, 类似傅克反应已有文献报道 ${ }^{[23-24]}$.
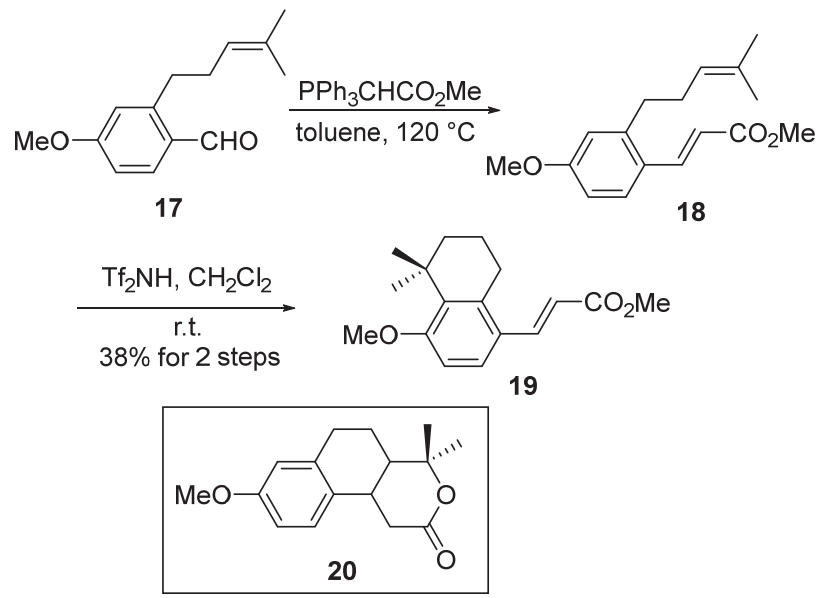

图式 5 化合物 18 的环加成反应尝试

Scheme 5 Attempt on cyclization of compound 18

考虑到分子内的 NHTs 作为最终的亲核试剂关环更 有利于环化反应的发生，在化合物 14 的结构上引入 NHTs 基团，设计了化合物 22，如 Scheme 6 所示. 化合 物 13 经过 Aldol 反应得到化合物 21, 后者发生差基消除 [25]得到化合物 22. 化合物 22 在 $\mathrm{Tf}_{2} \mathrm{NH}$ 的催化下依然没 有得到预期的环化产物，仅得到了双键发生傅克反应的 产物 23. 此外, 在 $\mathrm{Tf}_{2} \mathrm{NH}$ 的催化下化合物 21 反应完全 后即淬灭反应，以 $67 \%$ 的收率得到傅克反应产物 24 以 及 $10 \%$ 的收率得到傅克反应后羟基消除产物 23 , 该实 验结果说明傅克反应的速率快于羟基的消除反应.

以上实验结果表明，在相同的酸催化条件下，苯环 骨架的类似底物的缺电子双键并不能优先于另外孤立 双键形成茮位碳正离子, 而吲哚环骨架的存在可能是使 其优先形成苄位碳正离子所必须的. 为了进一步验证这 个设想，设计了含吲哚骨架的化合物 26, 如 Scheme 7 所 示.

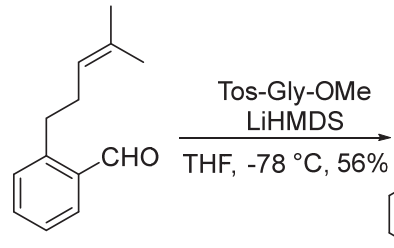

13<smiles>COC(N)C(O)C(O)c1ccccc1CCC=C(C)C</smiles>

21, $d r=1.5: 1$<smiles>[3H]N/C(=C\c1ccccc1CCCCC)C(OC)OC</smiles>

22

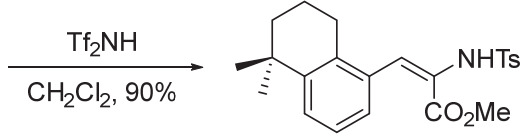

23

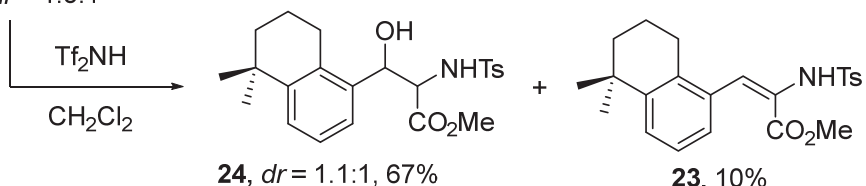

图式 6 化合物 22 的环加成反应尝试

Scheme 6 Attempt on cyclization of compound 22 


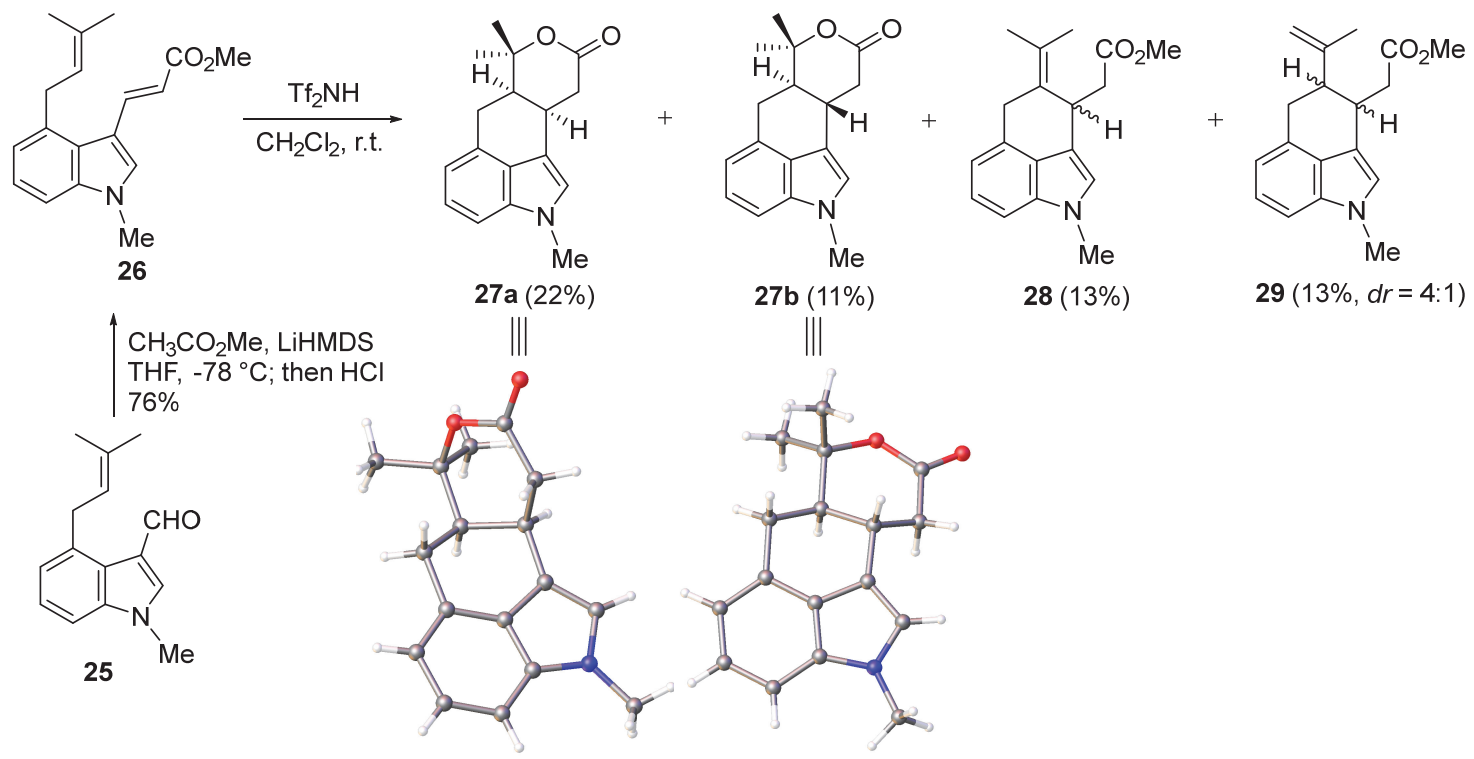

图式 7 化合物 26 的环加成反应尝试

Scheme 7 Attempt on cyclization of compound 26

从已知化合物 $\mathbf{2 5}^{[2]}$ 出发, 经过羟醛缩合和消除反应 即得到目标化合物 26. 化合物 $\mathbf{2 6}$ 在 $\mathrm{Tf}_{2} \mathrm{NH}$ 的催化下不 仅得到了四环内酯环化合物 $\mathbf{2 7 a}$ 和 $\mathbf{2 7 b}$, 同时还得到了 三环化合物 28 和 29 , 其中, 化合物 $27 \mathrm{a}$ 和 $27 \mathrm{~b}$ 的 C/D 并环的相对构型得到了单晶的进一步确认, 而得到的化 合物 29 是一对色谱难以分离的顺反异构体. 这个结果 说明, 在相同的条件下, 含有吲哚骨架的化合物 $\mathbf{2 6}$ 的缺 电子双键优先于孤立三取代双键而生成了茮位碳正离 子(可以与吲哚共振形成亚胺离子), 并引发了后续的环 化反应.

对这个反应过程进行了推测, 如 Scheme 8 所示: 质 子酸活化化合物 $\mathbf{2 6}$ 的羰基, 一方面, 由于吲哚氮原子的
共轭效应参与其中，使得缺电子不饱和酯双键更容易形 成芐位碳正离子(得到了比较稳定的芐位碳正离子), 另 一方面, 由于吲哚共振所形成的亚胺离子的活化作用, 使得形成的茮位碳正离子更容易引发烯烃的环化得到 3 级碳正离子中间体 Int-5. 如果 Int-5 的碳正离子被酯基 的羰基氧亲核进攻, 就能得到内酯环化合物 $27 \mathbf{a}$ 和 $27 \mathrm{~b}$; 如果 Int-5 发生质子消除, 就得到化合物 28 和 29 . 而吲 哚氮原子这样的双活化作用在苯环取代的衍生物中并 不存在, 因此在苯环衍生物 14、18 和 22 中, 缺电子不 饱和酯双键的活性不足以与临近的双键发生环化, 此时 更富电性的三取代孤立双键优先被质子化, 然后与芳环 发生傅克反应.<smiles>CC(=O)/C=C/c1cn(C)c2cccc(CC=C(C)C)c12</smiles><smiles>COC(O)=CCc1cn(C)c2cccc(CC=C(C)C)c12</smiles><smiles>COC(=O)C[C@H]1c2cn(C)c3cccc(c23)C[C@H]1C</smiles>

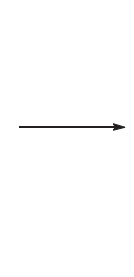<smiles>CC(=O)CC1C(=C(C)C)Cc2cccc3c2c1cn3C</smiles>

28

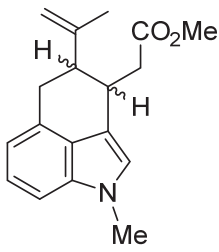

$29(d r=4: 1)$<smiles>C[C@H]1OC(=O)C[C@@H]2c3cccc4c3c(cn4C)C[C@@H]21</smiles>

27b

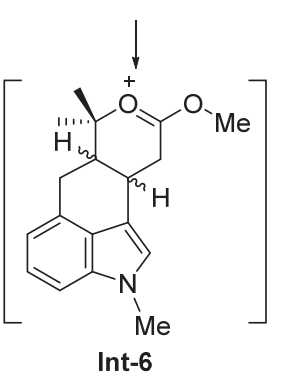

图式 $8[4+2]$ 环加成反应机理推测

Scheme 8 A possible mechanism for $[4+2]$ annulation 
上述实验结果也间接印证了我们所推测的两条途 径的反应机理中, path B 途径比 path A 途径更合理, 即 吲哚氮原子在吲哚茮位碳正离子引发的串联环化反应 过程中起到了关键作用.

\section{2 结论}

通过对在 $\alpha$-CPA 和 speradine $\mathrm{C}$ 合成中发展的吲哚 芐位碳正离子引发的仿生串联环化反应进行普适性研 究发现, 该芐位碳正离子串联环化反应在含有吲哚的底 物中能顺利发生, 说明吲哚氮原子的共轭效应在吲哚茮 位碳正阳离子串联环化反应中起到了关键性作用.

\section{3 实验部分}

\section{1 仪器与试剂}

所有试剂皆从试剂公司购得并直接使用. 实验所用 溶剂二氯甲烷、甲苯、四氢呋喃皆由溶剂纯化系统纯化 (Innovation 公司).

监测反应所用的薄层色谱硅胶铝板 (GF254) 由 Merck 公司所产; 显色剂使用磷钿酸、茴香酫及高锰酸 钾. 柱色谱使用青岛海洋化工厂生产的 200 300 目硅 胶; 制备薄层色谱使用烟台新诺化工有限公司所生产的 薄层层析硅胶板 $(200 \mathrm{~mm} \times 200 \mathrm{~mm} \times 1 \mathrm{~mm})$; 所用石油 醚、乙酸乙酯均购自北京市通广精细化工公司.

化合物高分辨质谱 ESI-MS 由 Waters Xevo G2 Q-TOF 型质谱仪测定; 熔点在 X-4 数字显示显微熔点测 定仪上测定(北京泰克仪器有限公司); 核磁共振氢谱由 Bruker Avance III $400 \mathrm{M}$ 或 Bruker Avance III $600 \mathrm{M}$ 核磁 共振谱仪测定, 核磁共振碳谱由 Bruker Avance III 100 $\mathrm{M}$ 或 Bruker Avance III $150 \mathrm{M}$ 核磁共振谱仪测定, 核磁 化学位移根据所用的溶剂 $\mathrm{CDCl}_{3}(\delta 7.26$ 和 77.0)进行定 标.

\section{2 实验方法}

3.2.1 3-羟基-3-(2-(3-甲基丁-2-烯-1-基)苯基)-2-((4甲基苯基)磺酰胺基)丙酸甲酯(10)的合成

氩气保护下, 将 2-(4-甲基苯基磺酰氨基)乙酸甲酯 (Tos-Gly-OMe, $838 \mathrm{mg}, 3.45 \mathrm{mmol}$ )溶于干燥四氢呋喃 (THF)溶液 $(20 \mathrm{~mL})$, 降温至 $-78{ }^{\circ} \mathrm{C}$, 缓慢滴加 LiHMDS 溶液 $(1.30 \mathrm{~mol} / \mathrm{L}$ in THF, $5.30 \mathrm{~mL}, 6.89 \mathrm{mmol}) .-78{ }^{\circ} \mathrm{C}$ 下反应 $10 \mathrm{~min}$ 后, 将化合物 $9(600 \mathrm{mg}, 3.49 \mathrm{mmol})$ 的 THF 溶液 $(10 \mathrm{~mL}$ ) 滴加入上述反应液, 然后缓慢升至室 温继续摚拌反应, 薄层色谱(TLC)监测显示原料消耗完 全后, 缓慢加入饱和 $\mathrm{NH}_{4} \mathrm{Cl}$ 溶液 $(10 \mathrm{~mL})$. 反应液用乙酸 乙酯 $(30 \mathrm{~mL} \times 3)$ 萃取, 合并有机相并用饱和氯化钠水溶 液 $(20 \mathrm{~mL} \times 3)$ 洗涤, 无水硫酸钠干燥. 减压旋蒸除去溶 剂后所得残留物用硅胶柱色谱 $[V$ (石油醚)： $V($ 乙酸乙
酯) $=8:$ 1]分离纯化得到 $647 \mathrm{mg}$ 无色油状液体 10, 产 率 45\%. ${ }^{1} \mathrm{H}$ NMR $\left(\mathrm{CDCl}_{3}, 400 \mathrm{MHz}\right) \delta: 7.63 \sim 7.61(\mathrm{~m}$, $2 \mathrm{H}), 7.29 \sim 7.28(\mathrm{~m}, 1 \mathrm{H}), 7.23 \sim 7.20(\mathrm{~m}, 3 \mathrm{H}), 7.16 \sim 7.14$ $(\mathrm{m}, 2 \mathrm{H}), 5.68 \sim 5.65(\mathrm{~d}, J=9.6 \mathrm{~Hz}, 1 \mathrm{H}), 5.22 \sim 5.20$ (t, $J=$ $4.8 \mathrm{~Hz}, 1 \mathrm{H}), 5.18 \sim 5.14(\mathrm{~m}, 1 \mathrm{H}), 4.19 \sim 4.16(\mathrm{dd}, J=9.6$, $5.2 \mathrm{~Hz}, 1 \mathrm{H}), 3.35(\mathrm{~s}, 3 \mathrm{H}), 3.30 \sim 3.28(\mathrm{~d}, J=6.8 \mathrm{~Hz}, 2 \mathrm{H})$, $2.81 \sim 2.80(\mathrm{~m}, 1 \mathrm{H}), 2.40(\mathrm{~s}, 3 \mathrm{H}), 1.76(\mathrm{~s}, 3 \mathrm{H}), 1.74(\mathrm{~s}$, $3 \mathrm{H}) ;{ }^{13} \mathrm{C} \mathrm{NMR}\left(\mathrm{CDCl}_{3}, 100 \mathrm{MHz}\right) \delta: 170.0,143.6,138.7$, $136.5,136.5,133.4,129.6,129.5,128.3,127.2,126.2$, $125.7,122.7,71.2,60.1,52.1,31.2,25.7,21.5,17.9$. HRMS calcd for $\mathrm{C}_{22} \mathrm{H}_{28} \mathrm{NO}_{5} \mathrm{~S}[\mathrm{M}+\mathrm{H}]^{+}$418.1683, found 418.1682 .

3.2.2 2-(3,3-二甲基-1,3,4,5-四氢苯并 [c]氧杂环丁-1基)-2-((4-甲基苯基)磺酰胺基)乙酸甲酯(11)的合成

氩气保护下，将化合物 $\mathbf{1 0}(100 \mathrm{mg}, 0.240 \mathrm{mmol})$ 溶 于无水 $\mathrm{CH}_{2} \mathrm{Cl}_{2}$ 溶液 $(10 \mathrm{~mL})$ 中, 室温下加入 $\mathrm{Tf}_{2} \mathrm{NH}(15.0$ $\mathrm{mg}, 0.0534 \mathrm{mmol}$ ). 搅拌反应至 TLC 监测原料消耗完全, 加入饱和 $\mathrm{NaHCO}_{3}$ 溶液 $(10 \mathrm{~mL})$, 用二氯甲烷 $(10 \mathrm{~mL} \times 3)$ 萃取后, 合并有机相后用饱和氯化钠水溶液 $(10 \mathrm{~mL} \times 3)$ 洗涤, 无水硫酸钠干燥. 减压旋蒸除去溶剂, 所得残留 物用薄层色谱 $[V$ (石油醚)： $V($ 乙酸乙酯 $)=2: 1$ ]分离纯 化得到 $76 \mathrm{mg}$ 黄色油状液体 11, 产率 $75 \%$. ${ }^{1} \mathrm{H}$ NMR $\left(\mathrm{CDCl}_{3}, 400 \mathrm{MHz}\right) \delta: 7.77 \sim 7.75(\mathrm{~m}, 2 \mathrm{H}), 7.31 \sim 7.27(\mathrm{~m}$, $3 \mathrm{H}), 7.16 \sim 7.14(\mathrm{~m}, 2 \mathrm{H}), 7.09 \sim 7.07(\mathrm{~m}, 1 \mathrm{H}), 5.33 \sim 5.30$ (d, $J=10.0 \mathrm{~Hz}, 1 \mathrm{H}), 4.99 \sim 4.97(\mathrm{~d}, J=6.4 \mathrm{~Hz}, 1 \mathrm{H})$, $4.55 \sim 4.51(\mathrm{dd}, J=10.0,6.4 \mathrm{~Hz}, 1 \mathrm{H}), 3.36(\mathrm{~s}, 3 \mathrm{H}), 2.90 \sim$ $2.75(\mathrm{~m}, 2 \mathrm{H}), 2.43(\mathrm{~s}, 3 \mathrm{H}), 1.70 \sim 1.67(\mathrm{dd}, J=6.0,5.2 \mathrm{~Hz}$, $2 \mathrm{H}), 1.21(\mathrm{~s}, 3 \mathrm{H}), 1.11(\mathrm{~s}, 3 \mathrm{H}) ;{ }^{13} \mathrm{C} \mathrm{NMR}\left(\mathrm{CDCl}_{3}, 100\right.$ MHz) $\delta: 170.6,143.8,142.2,137.4,136.5,129.6,129.5$, $127.6,127.5,126.1,125.8,76.3,74.0,59.1,52.0,39.8$, $30.9,30.1,23.7,21.5$. HRMS calcd for $\mathrm{C}_{22} \mathrm{H}_{28} \mathrm{NO}_{5} \mathrm{~S}[\mathrm{M}+$ $\mathrm{H}]^{+} 418.1683$, found 418.1686 .

3.2.3 (E)-3-(5,5-二甲基-5,6,7,8-四氢菜-1-基)丙烯酸 甲酯(15)的合成

氩气保护下, 将化合物 $\mathbf{1 3}$ (300 mg, $1.59 \mathrm{mmol})$ 溶于 无水 $\mathrm{PhMe}$ 溶液 $(20 \mathrm{~mL})$ 中, 加入三苯基膦烯基乙酸甲酯 $(1.07 \mathrm{~g}, 3.19 \mathrm{mmol})$, 加热至 $120{ }^{\circ} \mathrm{C}$ 搅拌反应 $10 \mathrm{~h}$, 减压 浓缩, 重结晶除去不溶性杂质, 得到黄色油状液体粗产 物 14, 直接用于下一步反应.

氩气保护下, 将粗产物化合物 14 溶于无水 $\mathrm{CH}_{2} \mathrm{Cl}_{2}$ 溶液 $(30 \mathrm{~mL})$ 中, 搅拌下加入 $\mathrm{Tf}_{2} \mathrm{NH}(71.7 \mathrm{mg}, 0.255$ $\mathrm{mmol}$ ). 室温下反应 $30 \mathrm{~min}$, 加入饱和 $\mathrm{NaHCO}_{3}$ 溶液(15 $\mathrm{mL})$, 分离有机相, 水相用二氯甲烷 $(20 \mathrm{~mL} \times 3)$ 萃取, 合 并有机相后用饱和氯化钠水溶液 $(10 \mathrm{~mL} \times 3)$ 洗涤, 无水 硫酸钠干燥. 减压旋蒸除去溶剂, 所得残留物用薄层色 
谱 $[V$ (石油醚)： $V($ 乙酸乙酯 $)=10 ： 1]$ 分离纯化得到 $249 \mathrm{mg}$ 黄色油状液体 15, 两步收率 64\%. ${ }^{1} \mathrm{H}$ NMR $\left(\mathrm{CDCl}_{3}, 400 \mathrm{MHz}\right) \delta: 8.04 \sim 8.00(\mathrm{~d}, J=15.6 \mathrm{~Hz}, 1 \mathrm{H})$, $7.42 \sim 7.40(\mathrm{~d}, J=7.6 \mathrm{~Hz}, 1 \mathrm{H}), 7.36 \sim 7.34(\mathrm{~d}, J=7.2 \mathrm{~Hz}$, $1 \mathrm{H}), 7.20 \sim 7.16(\mathrm{t}, J=7.6 \mathrm{~Hz}, 1 \mathrm{H}), 6.33 \sim 6.29(\mathrm{~d}, J=$ $15.6 \mathrm{~Hz}, 1 \mathrm{H}), 3.81(\mathrm{~s}, 3 \mathrm{H}), 2.86 \sim 2.83(\mathrm{t}, J=6.4 \mathrm{~Hz}, 2 \mathrm{H})$, $1.88 \sim 1.82(\mathrm{~m}, 2 \mathrm{H}), 1.68 \sim 1.65(\mathrm{~m}, 2 \mathrm{H}), 1.30(\mathrm{~s}, 6 \mathrm{H}) ;{ }^{13} \mathrm{C}$ NMR $\left(\mathrm{CDCl}_{3}, 100 \mathrm{MHz}\right) \delta: 167.4,146.6,143.3,135.4$, 133.5, 128.7, 125.8, 123.9, 119.2, 51.6, 38.4, 34.0, 31.9, 27.7, 19.2. HRMS calcd for $\mathrm{C}_{16} \mathrm{H}_{21} \mathrm{O}_{2}[\mathrm{M}+\mathrm{H}]^{+} 245,1536$, found 245.1537).

3.2.4 (E)-3-(4-甲氧基-5,5-二甲基-5,6,7,8-四氢菜-1基)丙烯酸甲酯(19)的合成

氩气保护下, 将化合物 $\mathbf{1 7}(300 \mathrm{mg}, 1.37 \mathrm{mmol}$ ) 溶于 无水 $\mathrm{PhMe}$ 溶液 $(20 \mathrm{~mL}$ )中, 加入三苯基膦烯基乙酸甲酯 $(920 \mathrm{mg}, 2.75 \mathrm{mmol})$, 加热至 $120{ }^{\circ} \mathrm{C}$ 摚拌反应 $10 \mathrm{~h}$, 减压 浓缩后, 重结晶除去不溶性杂质, 得到黄色油状液体粗 产物 18, 直接用于下一步反应.

氞气保护下, 将粗产物化合物 18 溶于无水 $\mathrm{CH}_{2} \mathrm{Cl}_{2}$ 溶液 $(30 \mathrm{~mL})$ 中, 搅拌下加入 $\mathrm{Tf}_{2} \mathrm{NH}(49.5 \mathrm{mg}, 0.176$ $\mathrm{mmol})$. 室温下反应 $30 \mathrm{~min}$, 加入饱和 $\mathrm{NaHCO}_{3}$ 溶液(15 $\mathrm{mL})$, 分离有机相. 水相用二氯甲烷 $(20 \mathrm{~mL} \times 3)$ 萃取, 合 并有机相后用饱和氯化钠水溶液 $(10 \mathrm{~mL} \times 3)$ 洗涤, 无水 硫酸钠干燥. 减压旋蒸除去溶剂, 所得残留物用薄层色 谱 $[V($ 石油醚 $): V($ 乙酸乙酯 $)=10: 1]$ 分离纯化得到 $144 \mathrm{mg}$ 黄色油状液体 19, 两步收率 $38 \% .{ }^{1} \mathrm{H}$ NMR $\left(\mathrm{CDCl}_{3}, 400 \mathrm{MHz}\right) \delta: 8.00 \sim 7.96(\mathrm{~d}, J=15.6 \mathrm{~Hz}, 1 \mathrm{H})$, $7.40 \sim 7.38(\mathrm{~d}, J=8.4 \mathrm{~Hz}, 1 \mathrm{H}), 6.75 \sim 6.73(\mathrm{~d}, J=8.4 \mathrm{~Hz}$, $1 \mathrm{H}), 6.23 \sim 6.19(\mathrm{~d}, J=15.6 \mathrm{~Hz}, 1 \mathrm{H}), 3.84(\mathrm{~s}, 3 \mathrm{H}), 3.79$ (s, $3 \mathrm{H}), 2.83 \sim 2.79(\mathrm{t}, J=6.4 \mathrm{~Hz}, 2 \mathrm{H}), 1.79 \sim 1.73(\mathrm{~m}, 2 \mathrm{H})$, $1.63 \sim 1.60(\mathrm{~m}, 2 \mathrm{H}), 1.37(\mathrm{~s}, 6 \mathrm{H}) ;{ }^{13} \mathrm{C} \mathrm{NMR}\left(\mathrm{CDCl}_{3}, 100\right.$ MHz) $\delta: 167.8,160.7,143.3,138.3,134.3,126.4,125.3$, 116.9, 109.2, 55.0, 51.5, 41.9, 34.2, 29.1, 28.3, 19.1. HRMS calcd for $\mathrm{C}_{17} \mathrm{H}_{23} \mathrm{O}_{3}[\mathrm{M}+\mathrm{H}]^{+}$275.1642, found 275.1645 .

3.2.5 3-羟基-3-(2-(4-甲基戊-3-烯-1-基)苯基)-2-((4甲基苯基)磺酰胺基)丙酸甲酯(21)的合成

氩气保护下, 将 2-(4-甲基苯基磺酰氨基)乙酸甲酯 (Tos-Gly-OMe, 1.27 g, 5.23 mmol)溶于干燥 THF 溶液(15 $\mathrm{mL})$, 降温至 $-78{ }^{\circ} \mathrm{C}$, 缓慢滴加 LiHMDS 溶液(1.30 $\mathrm{mol} \cdot \mathrm{L}^{-1}$ in THF, $\left.4.43 \mathrm{~mL}, 5.75 \mathrm{mmol}\right) .-78{ }^{\circ} \mathrm{C}$ 下反应 10 $\min$ 后, 将化合物 $\mathbf{1 3}$ (492 $\mathrm{mg}, 2.62 \mathrm{mmol}$ )的 THF 溶液(5 $\mathrm{mL}$ )滴加入上述反应液, 然后缓慢升至室温继续傥拌反 应, TLC 监测原料消耗完全, 缓慢加入饱和 $\mathrm{NH}_{4} \mathrm{Cl}$ 溶液 $(10 \mathrm{~mL})$. 反应液用乙酸乙酯 $(30 \mathrm{~mL} \times 3)$ 萃取, 合并有机
相并用饱和氯化钠水溶液 $(20 \mathrm{~mL} \times 3)$ 洗涤, 无水硫酸钠 干燥. 减压旋蒸除去溶剂, 所得残留物用硅胶柱色谱 [ $V$ (石油醚) $: V$ (乙酸乙酯 $)=2: 1$ ] 分离纯化得到 $635 \mathrm{mg}$ 淡黄色油状液体 21 , 产率 $56 \%$. ${ }^{1} \mathrm{H}$ NMR $\left(\mathrm{CDCl}_{3}, 400\right.$ MHz) $\delta: 7.68 \sim 7.66(\mathrm{~d}, J=8.0 \mathrm{~Hz} 3 \mathrm{H}), 7.45 \sim 7.43$ (d, $J=$ $8.0 \mathrm{~Hz} 3 \mathrm{H}), 7.29 \sim 7.27(\mathrm{~m}, 2 \mathrm{H}), 7.25 \sim 7.20(\mathrm{~m}, 4 \mathrm{H})$, $7.19 \sim 7.15(\mathrm{~m}, 4 \mathrm{H}), 7.13 \sim 7.10(\mathrm{~m}, 4 \mathrm{H}), 5.76$ (brs, $1 \mathrm{H})$, 5.63 (brs, $1 \mathrm{H}), 5.35 \sim 5.33(\mathrm{t}, J=3.2 \mathrm{~Hz}, 1 \mathrm{H}), 5.26 \sim 5.23$ (t, $J=4.8 \mathrm{~Hz}, 1 \mathrm{H}), 5.16 \sim 5.13(\mathrm{~m}, 2 \mathrm{H}), 4.20 \sim 4.16(\mathrm{dd}, J$ $=9.6,4.4 \mathrm{~Hz}, 1 \mathrm{H}), 4.09 \sim 4.05(\mathrm{dd}, J=10.0,3.6 \mathrm{~Hz}, 1 \mathrm{H})$, $3.56(\mathrm{~s}, 3 \mathrm{H}), 3.33(\mathrm{~s}, 3 \mathrm{H}), 2.89 \sim 2.84(\mathrm{~m}, 1 \mathrm{H}), 2.64 \sim 2.59$ (m, 3H), 2.54 2.49 (m, 3H), $2.40(\mathrm{~s}, 3 \mathrm{H}), 2.36(\mathrm{~s}, 3 \mathrm{H})$, $2.26 \sim 2.19(\mathrm{~m}, 5 \mathrm{H}), 1.69(\mathrm{~s}, 6 \mathrm{H}), 1.53(\mathrm{~s}, 6 \mathrm{H}) ;{ }^{13} \mathrm{C} \mathrm{NMR}$ $\left(\mathrm{CDCl}_{3}, 100 \mathrm{MHz}\right) \delta: 170.7,169.8,143.7,143.2,139.0$, 138.6, 136.5, 136.2, 132.8, 132.6, 129.6, 129.5, 129.3, $129.2,128.1,128.1,127.2,126.9,126.6,126.1,126.0$, $125.5,123.2,123.1,71.3,70.3,60.8,60.2,52.7,52.1,31.9$, 31.8, 29.7, 29.6, 25.6, 21.5, 21.4, 17.6, 17.5. HRMS calcd for $\mathrm{C}_{23} \mathrm{H}_{29} \mathrm{NNaO}_{5} \mathrm{~S}[\mathrm{M}+\mathrm{Na}]^{+} 454.1659$, found 454.1657.

3.2.6 (E)-3-(2-(4-甲基戊-3-烯-1-基)苯基)-2-((4-甲基 苯基)磺酰胺基)丙烯酸甲酯(22)的合成

氩气保护下, 将化合物 $\mathbf{2 1}(300 \mathrm{mg}, 0.695 \mathrm{mmol})$ 溶 于 $10 \mathrm{~mL}$ 无水 $\mathrm{MeCN}$ 溶液中, 再向其加入 DMAP ( 8.50 $\mathrm{mg}, 0.0695 \mathrm{mmol})$ 和 $\mathrm{Boc}_{2} \mathrm{O}(0.162 \mathrm{ml}, 0.695 \mathrm{mmol})$. 搅拌 反应至 TLC 监测原料消耗完全, 再向反应液中加入四 甲基胍( TMG， $0.200 \mathrm{~mL}, 2 \%$ in volume). 继续反应至 TLC 监测原料消耗完全, 然后向反应液中加入 $\mathrm{KHSO}_{4}$ 溶液 $\left(1 \mathrm{~mol} \cdot \mathrm{L}^{-1}, 5 \mathrm{~mL}\right)$, 用乙酸乙酯 $(10 \mathrm{~mL} \times 3)$ 萃取, 合 并有机相，依次用 $\mathrm{KHSO}_{4}$ 溶液 $(5 \mathrm{~mL})$ 、饱和 $\mathrm{NaHCO}_{3}$ 溶 液 $(5 \mathrm{~mL})$ 、饱和氯化钠水溶液 $(5 \mathrm{~mL})$ 洗涤, 无水硫酸钠 干燥. 减压旋蒸除去溶剂, 所得残留物用薄层色谱 $[V$ (石 油醚)： $V$ (乙酸乙酯) $=2: 1$ ]分离纯化得到 $209 \mathrm{mg}$ 无色 油状液体 22, 产率 $73 \%$. ${ }^{1} \mathrm{H} \mathrm{NMR}\left(\mathrm{CDCl}_{3}, 400 \mathrm{MHz}\right) \delta$ : $7.66 \sim 7.64(\mathrm{~d}, J=8.0 \mathrm{~Hz}, 1 \mathrm{H}), 7.61(\mathrm{~s}, 1 \mathrm{H}), 7.59 \sim 7.57$ (d, $J=8.0 \mathrm{~Hz}, 2 \mathrm{H}), 7.25 \sim 7.21(\mathrm{td}, J=7.6,1.6 \mathrm{~Hz}, 1 \mathrm{H})$, $7.18 \sim 7.16(\mathrm{~m}, 3 \mathrm{H}), 7.12 \sim 7.08(\mathrm{td}, J=7.6,1.2 \mathrm{~Hz}, 1 \mathrm{H})$, 6.20 (s, $1 \mathrm{H}), 5.20 \sim 5.17$ (tt, $J=7.2,1.2 \mathrm{~Hz}, 1 \mathrm{H}), 3.67$ (s, $3 \mathrm{H}), 2.64 \sim 2.60(\mathrm{dd}, J=9.6,7.6 \mathrm{~Hz}, 2 \mathrm{H}), 2.38(\mathrm{~s}, 3 \mathrm{H})$, $2.28 \sim 2.22$ (dd, $J=15.6,7.6 \mathrm{~Hz}, 2 \mathrm{H}), 1.70$ (s, 3H), 1.58 (s, $3 \mathrm{H}) ;{ }^{13} \mathrm{C}$ NMR $\left(\mathrm{CDCl}_{3}, 100 \mathrm{MHz}\right) \delta: 165.3,143.6,142.0$, $136.8,133.1,132.6,131.4,129.5,129.3,129.3,129.2$, 127.3, 126.0, 124.7, 123.3, 52.6, 33.9, 29.5, 25.7, 21.5, 17.5. HRMS calcd for $\mathrm{C}_{23} \mathrm{H}_{28} \mathrm{NO}_{4} \mathrm{~S}[\mathrm{M}+\mathrm{H}]^{+} 414.1734$, found 414.1735 . 
3.2.7 (E)-3-(5,5-二甲基-5,6,7,8-四氢菜-1-基)-2-((4甲基苯基)磺酰胺基)丙烯酸甲酯(23)的合成

氩气保护下, 将化合物 $22(100 \mathrm{mg}, 0.236 \mathrm{mmol})$ 溶 于无水 $\mathrm{CH}_{2} \mathrm{Cl}_{2}$ 溶液 $(10 \mathrm{~mL})$ 中, 室温下加入 $\mathrm{Tf}_{2} \mathrm{NH}(13.3$ $\mathrm{mg}, 0.0472 \mathrm{mmol}$ ). 搅拌反应至 TLC 监测原料消耗完全, 加入饱和 $\mathrm{NaHCO}_{3}$ 溶液 $(10 \mathrm{~mL})$, 用二氯甲烷 $(10 \mathrm{~mL} \times 3)$ 萃取后, 合并有机相后用饱和氯化钠水溶液 $(10 \mathrm{~mL} \times 3)$ 洗涤, 无水硫酸钠干燥. 减压旋蒸除去溶剂, 所得残留 物用制备薄层色谱 $[V$ (石油醚)： $V($ 乙酸乙 酯 $)=2: 1]$ 分离纯化得到 $90 \mathrm{mg}$ 无色油状液体 23, 产率 $90 \%$. ${ }^{1} \mathrm{H}$ NMR $\left(\mathrm{CDCl}_{3}, 400 \mathrm{MHz}\right) \delta: 7.53 \sim 7.53(\mathrm{~d}, J=8.4 \mathrm{~Hz}$, $2 \mathrm{H}), 7.45(\mathrm{~s}, 1 \mathrm{H}), 7.29 \sim 7.27(\mathrm{~d}, J=8.8 \mathrm{~Hz}, 1 \mathrm{H}), 7.20 \sim$ $7.18(\mathrm{~d}, J=7.6 \mathrm{~Hz}, 1 \mathrm{H}), 7.16 \sim 7.14(\mathrm{~d}, J=8.0 \mathrm{~Hz}, 2 \mathrm{H})$, $7.01 \sim 6.97(\mathrm{dd}, J=8.0,7.6 \mathrm{~Hz}, 1 \mathrm{H}), 6.26(\mathrm{~s}, 1 \mathrm{H}), 3.72(\mathrm{~s}$, $3 \mathrm{H}), 2.62 \sim 2.59(\mathrm{t}, J=6.4 \mathrm{~Hz}, 1 \mathrm{H}), 2.38(\mathrm{~s}, 3 \mathrm{H}), 1.81 \sim$ $1.78(\mathrm{~m}, 2 \mathrm{H}), 1.64 \sim 1.61(\mathrm{~m}, 2 \mathrm{H}), 1.28(\mathrm{~s}, 6 \mathrm{H}) ;{ }^{13} \mathrm{C} \mathrm{NMR}$ $\left(\mathrm{CDCl}_{3}, 100 \mathrm{MHz}\right) \delta$ : 165.4, 146.2, 143.4, 137.1, 135.5, $133.2,131.7,129.2,127.8,127.2,126.0,125.6,125.2$, 52.7, 38.5, 34.0, 31.8, 28.2, 21.6, 19.2. HRMS calcd for $\mathrm{C}_{23} \mathrm{H}_{28} \mathrm{NO}_{4} \mathrm{~S}[\mathrm{M}+\mathrm{H}]^{+}$414.1734, found 414.1732.

3.2.8 3-(5,5-二甲基-5,6,7,8-四氢荟-1-基)-3-羟基2-((4-甲基苯基)磺酰胺基)丙酸甲酯(24)的合成

氩气保护下, 将化合物 21 (100 mg, $0.232 \mathrm{mmol}$ )溶 于无水 $\mathrm{CH}_{2} \mathrm{Cl}_{2}$ 溶液 $(10 \mathrm{~mL})$ 中, 室温下加入 $\mathrm{Tf}_{2} \mathrm{NH}(13.0$ $\mathrm{mg}, 0.0463 \mathrm{mmol}$ ). 搅拌反应至 TLC 监测原料消耗完全, 加入饱和 $\mathrm{NaHCO}_{3}$ 溶液 $(10 \mathrm{~mL})$, 用二氯甲烷 $(10 \mathrm{~mL} \times 3)$ 萃取后, 合并有机相后用饱和氯化钠水溶液 $(10 \mathrm{~mL} \times 3)$ 洗涤, 无水硫酸钠干燥. 减压旋蒸除去溶剂, 所得残留 物用制备薄层色谱 $[V$ (石油醚)： $V($ 乙酸乙 酯 $)=2: 1]$ 分离纯化得到 $67 \mathrm{mg}$ 无色油状液体 24, 产率 $67 \%$. ${ }^{1} \mathrm{H}$ NMR $\left(\mathrm{CDCl}_{3}, 400 \mathrm{MHz}\right) \delta: 7.70 \sim 7.68(\mathrm{~d}, \quad J=8.0 \mathrm{~Hz}$, $2 \mathrm{H}), 7.39 \sim 7.37(\mathrm{~d}, J=8.4 \mathrm{~Hz}, 2 \mathrm{H}), 7.30 \sim 7.24(\mathrm{~m}, 5 \mathrm{H})$, $7.11 \sim 7.06$ (m, 5H), 5.71 (brs, 1H), 5.53 (brs, 1H), 5.31 $(\mathrm{m}, 1 \mathrm{H}), 5.19 \sim 5.17(\mathrm{t}, J=4.8 \mathrm{~Hz}, 1 \mathrm{H}), 4.18 \sim 4.15(\mathrm{dd}$, $J=10.0,4.8 \mathrm{~Hz}, 1 \mathrm{H}), 4.09 \sim 4.06(\mathrm{dd}, J=10.0,2.8 \mathrm{~Hz}$, $1 \mathrm{H}), 3.63(\mathrm{~s}, 3 \mathrm{H}), 3.32(\mathrm{~s}, 3 \mathrm{H}), 2.73 \sim 2.64(\mathrm{~m}, 3 \mathrm{H}), 2.47 \sim$ $2.43(\mathrm{~m}, 3 \mathrm{H}), 2.40(\mathrm{~s}, 3 \mathrm{H}), 2.34(\mathrm{~s}, 3 \mathrm{H}), 1.80 \sim 1.75(\mathrm{~m}$, $4 \mathrm{H}), 1.64 \sim 1.63(\mathrm{~m}, 4 \mathrm{H}), 1.29(\mathrm{~s}, 3 \mathrm{H}), 1.26 \sim 1.25(\mathrm{~m}$, $9 \mathrm{H}) ;{ }^{13} \mathrm{C}$ NMR $\left(\mathrm{CDCl}_{3}, 100 \mathrm{MHz}\right) \delta: 170.9,169.7,146.2$, $145.9,143.7,143.1,136.7,136.6,136.4,136.4,132.9$, $132.3,129.6,129.3,127.3,127.0,126.9,126.7,125.8$, 125.6, 123.6, 122.5, 71.4, 70.2, 59.8, 59.3, 52.7, 52.0, 38.5, $34.1,34.0,32.1,32.1,26.7,26.6,21.5,21.5,19.4,19.3$. HRMS calcd for $\mathrm{C}_{23} \mathrm{H}_{29} \mathrm{NNaO}_{5} \mathrm{~S}[\mathrm{M}+\mathrm{Na}]^{+}$454.1659, found 454.1670 .
3.2.9 (E)-3-(1-甲基-4-(3-甲基丁-2-烯-1-基)- $1 H$-吲 哚-3-基)丙烯酸甲酯(26)的合成

氩气保护下, 将化合物乙酸甲酯 $(0.780 \mathrm{~mL}, 9.69$ mmol)溶于无水 THF 溶液 $(50 \mathrm{~mL})$ 中, 降温至 $-78{ }^{\circ} \mathrm{C}$, 缓慢滴加 LiHMDS 溶液 $\left(1.30 \mathrm{~mol} \cdot \mathrm{L}^{-1}\right.$ in THF, $8.13 \mathrm{~mL}$, $10.6 \mathrm{mmol}$ ). $-78{ }^{\circ} \mathrm{C}$ 下搅拌 $10 \mathrm{~min}$ 后, 将化合物 $\mathbf{2 5}$ 的 THF 溶液 $\left(0.881 \mathrm{~mol} \cdot \mathrm{L}^{-1}\right.$ in THF, $\left.10 \mathrm{~mL}, 8.81 \mathrm{mmol}\right)$ 滴加 入上述反应液, 然后缓慢升至室温继续反应, TLC 监测 原料消耗完全, 缓慢滴加 $4 \mathrm{~mol} \cdot \mathrm{L}^{-1} \mathrm{HCl}$ 水溶液, 至溶 液中白色沉淀消失为止. 反应液用乙酸乙酯 $(50 \mathrm{~mL} \times 3)$ 萃取, 合并有机相后用饱和氯化钠水溶液 $(30 \mathrm{~mL} \times 3)$ 洗 涤, 无水硫酸钠干燥. 减压旋蒸除去溶剂, 所得残留物 用硅胶柱色谱 $[V$ (石油醚)： $V($ 乙酸乙酯 $)=15: 1$ ]纯化 得到 $1.88 \mathrm{~g}$ 白色粉末状固体 26, 产率 76\%. m.p. 93.7 $94.2{ }^{\circ} \mathrm{C} ;{ }^{1} \mathrm{H}$ NMR $\left(\mathrm{CDCl}_{3}, 400 \mathrm{MHz}\right) \delta: 8.23 \sim 8.19(\mathrm{~d}$, $J=16.0 \mathrm{~Hz}, 1 \mathrm{H}), 7.47$ (s, 1H), $7.20 \sim 7.19$ (d, $J=4.0 \mathrm{~Hz}$, $2 \mathrm{H}), 7.02 \sim 7.00(\mathrm{t}, J=4.0 \mathrm{~Hz}, 1 \mathrm{H}), 6.20 \sim 6.16(\mathrm{~d}, J=$ $16.0 \mathrm{~Hz}, 1 \mathrm{H}), 5.37 \sim 5.34(\mathrm{t}, J=6.8 \mathrm{~Hz}, 1 \mathrm{H}), 3.80(\mathrm{~s}, 3 \mathrm{H})$, $3.79(\mathrm{~m}, 5 \mathrm{H}), 1.81$ (s, $3 \mathrm{H}), 1.77$ (s, $3 \mathrm{H}) ;{ }^{13} \mathrm{C}$ NMR $\left(\mathrm{CDCl}_{3}\right.$, $100 \mathrm{MHz}) \delta: 168.1,139.7,137.8,135.2,133.3,128.5$, $125.1,122.6,122.5,121.4,112.7,112.5,107.9,51.3,33.3$, 32.9, 25.7, 18.1. HRMS calcd for $\mathrm{C}_{18} \mathrm{H}_{22} \mathrm{NO}_{2}[\mathrm{M}+\mathrm{H}]^{+}$ 284.1645 , found 284.1650 .

\section{2 .10 化合物 $27 \mathrm{a} 、 27 \mathrm{~b} 、 28 、 29$ 的合成}

氩气保护下, 将化合物 $\mathbf{2 6}(100 \mathrm{mg}, 0.353 \mathrm{mmol})$ 溶 于无水 $\mathrm{CH}_{2} \mathrm{Cl}_{2}$ 溶液 $(10 \mathrm{~mL})$ 中, 搅拌下加入 $\mathrm{Tf}_{2} \mathrm{NH}(19.9$ $\mathrm{mg}, 0.0706 \mathrm{mmol})$. 室温下反应 $30 \mathrm{~min}$ 后, 加入饱和 $\mathrm{NaHCO}_{3}$ 溶液 $(5 \mathrm{~mL})$, 反应液用二氯甲烷 $(10 \mathrm{~mL} \times 3)$ 萃 取, 合并有机相后用饱和氯化钠水溶液 $(10 \mathrm{~mL} \times 3)$ 洗涤, 无水硫酸钠干燥. 减压旋蒸除去溶剂, 所得残留物用薄 层色谱 $[V$ (石油醚) $: V($ 乙酸乙酯 $)=4: 1$ ]分离纯化得到 (6aS, $10 \mathrm{a} S)$-2,7,7-三甲基-2,6,6a,7,10,10a-六氢- $9 \mathrm{H}$-异色素 [5,6,7-cd]吲哚-9-酮(27a), 淡黄色固体 $21 \mathrm{mg}$, 产率 $22 \%$. m.p. $179.6 \sim 180.2{ }^{\circ} \mathrm{C} ;{ }^{1} \mathrm{H}$ NMR $\left(\mathrm{CDCl}_{3}, 400 \mathrm{MHz}\right) \delta$ : $7.22 \sim 7.18(\mathrm{dd}, J=8.0,6.8 \mathrm{~Hz}, 1 \mathrm{H}), 7.15 \sim 7.12(\mathrm{~d}, J=8.0$ $\mathrm{Hz}, 1 \mathrm{H}), 6.91 \sim 6.89(\mathrm{~d}, J=6.8 \mathrm{~Hz}, 1 \mathrm{H}), 3.86 \sim 3.80(\mathrm{~m}$, $1 \mathrm{H}), 3.78(\mathrm{~s}, 1 \mathrm{H}), 3.17 \sim 3.12$ (dd, $J=16.4,4.4 \mathrm{~Hz}, 1 \mathrm{H}$ ), $3.01 \sim 2.94(\mathrm{dd}, J=16.4,12.4 \mathrm{~Hz}, 1 \mathrm{H}), 2.82 \sim 2.76(\mathrm{dd}$, $J=18.8,6.4 \mathrm{~Hz}, 1 \mathrm{H}), 2.45 \sim 2.37(\mathrm{dd}, J=18.8,12.0 \mathrm{~Hz}$, $1 \mathrm{H}), 2.25 \sim 2.20$ (dt, $J=12.4,4.4 \mathrm{~Hz}, 1 \mathrm{H}), 1.61(\mathrm{~s}, 3 \mathrm{H})$, $1.57(\mathrm{~s}, 3 \mathrm{H}) ;{ }^{13} \mathrm{C} \mathrm{NMR}\left(\mathrm{CDCl}_{3}, 100 \mathrm{MHz}\right) \delta: 170.6,134.9$, 129.0, 125.6, 123.0, 122.2, 116.2, 114.5, 106.9, 84.0, 41.5, $34.5,32.8,29.7,27.2,26.9,24.2$. HRMS calcd for $\mathrm{C}_{17} \mathrm{H}_{20^{-}}$ $\mathrm{NO}_{2}[\mathrm{M}+\mathrm{H}]^{+}$270.1489, found 270.1487 .

(6aS,10a $R$ )-2,7,7-三甲基-2,6,6a,7,10,10a-六氢-9H-异 
色素[5,6,7-cd]吲哚-9-酮(27b): 淡黄色固体 $11 \mathrm{mg}$, 产率 11\%. m.p. $199.8 \sim 200.6{ }^{\circ} \mathrm{C} ;{ }^{1} \mathrm{H} \mathrm{NMR}\left(\mathrm{CDCl}_{3}, 400 \mathrm{MHz}\right)$ $\delta: 7.20 \sim 7.16(\mathrm{dd}, J=8.4,6.8 \mathrm{~Hz}, 1 \mathrm{H}), 7.15 \sim 7.13(\mathrm{~d}, J=$ $8.4 \mathrm{~Hz}, 1 \mathrm{H}), 6.87 \sim 6.86(\mathrm{~d}, J=6.8 \mathrm{~Hz}, 1 \mathrm{H}), 6.75(\mathrm{~s}, 1 \mathrm{H})$, $3.78(\mathrm{~s}, 3 \mathrm{H}), 3.44 \sim 3.37(\mathrm{~m}, 1 \mathrm{H}), 3.30 \sim 3.24(\mathrm{dd}, J=18.0$, $5.6 \mathrm{~Hz}, 1 \mathrm{H}), 3.09 \sim 3.04(\mathrm{dd}, J=15.2,4.0 \mathrm{~Hz}, 1 \mathrm{H}), 2.90 \sim$ $2.83(\mathrm{dd}, J=15.2,12.0 \mathrm{~Hz}, 1 \mathrm{H}), 2.57 \sim 2.50$ (dd, $J=18.0$, $12.0 \mathrm{~Hz}, 1 \mathrm{H}), 2.11 \sim 2.04(\mathrm{~m}, 1 \mathrm{H}), 1.60(\mathrm{~s}, 3 \mathrm{H}), 1.50(\mathrm{~s}$, $3 \mathrm{H}) ;{ }^{13} \mathrm{C} \mathrm{NMR}\left(\mathrm{CDCl}_{3}, 100 \mathrm{MHz}\right) \delta: 170.3,134.6,129.5$, 126.2, 122.9, 122.0, 115.6, 114.7, 107.0, 85.8, 46.8, 36.3, 32.9 , 29.5, 29.3, 29.1, 23.6. HRMS calcd for $\mathrm{C}_{17} \mathrm{H}_{20} \mathrm{NO}_{2}$ $[\mathrm{M}+\mathrm{H}]^{+}$270.1489, found 270.1487 .

2-(1-甲基-4-(丙烷-2-亚烷基)-1,3,4,5-四氢苯并 $[c d]$ 吲哚-3-基)乙酸甲酯(28): 淡黄色油状液体 $13 \mathrm{mg}$, 产率 $13 \% .{ }^{1} \mathrm{H}$ NMR $\left(\mathrm{CDCl}_{3}, 600 \mathrm{MHz}\right) \delta: 7.15 \sim 7.13$ (dd, $J=$ 8.4, 7.2 Hz, 1H), 7.07 7.05 (d, $J=8.4 \mathrm{~Hz}, 1 \mathrm{H}), 6.84 \sim$ $6.83(\mathrm{~d}, J=7.2 \mathrm{~Hz}, 1 \mathrm{H}), 6.76(\mathrm{~s}, 1 \mathrm{H}), 4.65 \sim 4.62(\mathrm{dd}, J=$ 9.0, $6.6 \mathrm{~Hz}, 1 \mathrm{H}), 3.94 \sim 3.91(\mathrm{~d}, J=18.0 \mathrm{~Hz}, 1 \mathrm{H}), 3.73$ (s, $3 \mathrm{H}), 3.67(\mathrm{~s}, 3 \mathrm{H}), 3.53 \sim 3.50(\mathrm{~d}, J=17.4 \mathrm{~Hz}, 1 \mathrm{H}), 2.62 \sim$ $2.55(\mathrm{~m}, 2 \mathrm{H}), 1.84 \sim 1.83(\mathrm{~d}, J=1.8 \mathrm{~Hz}, 3 \mathrm{H}), 1.80 \sim 1.80$ $(\mathrm{d}, J=0.6 \mathrm{~Hz}, 3 \mathrm{H}) ;{ }^{13} \mathrm{C} \mathrm{NMR}\left(\mathrm{CDCl}_{3}, 150 \mathrm{MHz}\right) \delta: 172.9$, $134.6,130.8,129.6,126.7,125.9,123.0,122.6,115.0$, $114.9,106.2,51.3,41.8,32.8,32.5,28.0,21.1,20.8$. HRMS calcd for $\mathrm{C}_{18} \mathrm{H}_{22} \mathrm{NO}_{2}[\mathrm{M}+\mathrm{H}]^{+}$284.1645, found 284.1649 .

2-(1-甲基-4-(丙-1-烯-2-基)-1,3,4,5-四氢苯并 $[\mathrm{cd}]$ 吲 哚-3-基)乙酸甲酯(29): 无色油状液 $13 \mathrm{mg}$, 产率 $13 \%$. ${ }^{1} \mathrm{H}$ NMR $\left(\mathrm{CDCl}_{3}, 600 \mathrm{MHz}\right) \delta: 7.16 \sim 7.12(\mathrm{dd}, J=8.4,6.6$ $\mathrm{Hz}, 1 \mathrm{H}), 7.09 \sim 7.07(\mathrm{~d}, J=8.4 \mathrm{~Hz}, 1 \mathrm{H}), 6.85 \sim 6.84(\mathrm{~d}$, $J=6.6 \mathrm{~Hz}, 1 \mathrm{H}), 6.81 \sim 6.80(\mathrm{~d}, J=7.2 \mathrm{~Hz}, 1 \mathrm{H}), 6.77(\mathrm{~s}$, $1 \mathrm{H}), 6.71(\mathrm{~s}, 1 \mathrm{H}), 5.00(\mathrm{~s}, 1 \mathrm{H}), 4.91(\mathrm{~s}, 1 \mathrm{H}), 4.90(\mathrm{~s}, 1 \mathrm{H})$, $4.83(\mathrm{~s}, 1 \mathrm{H}), 3.78 \sim 3.76(\mathrm{~m}, 1 \mathrm{H}), 3.75(\mathrm{~s}, 3 \mathrm{H}), 3.74(\mathrm{~s}$, $3 \mathrm{H}), 3.65(\mathrm{~s}, 3 \mathrm{H}), 3.51 \sim 3.47(\mathrm{~m}, 1 \mathrm{H}), 3.15 \sim 3.10(\mathrm{dd}, J=$ $11.4,10.2 \mathrm{~Hz}, 1 \mathrm{H}), 3.09 \sim 3.04(\mathrm{dd}, J=15.6,12.0 \mathrm{~Hz}, 1 \mathrm{H})$, $2.95 \sim 2.90(\mathrm{~m}, 1 \mathrm{H}), 2.83 \sim 2.80(\mathrm{dd}, J=15.6,3.6 \mathrm{~Hz}, 1 \mathrm{H})$, $2.77 \sim 2.74(\mathrm{dt}, J=12.0,3.6 \mathrm{~Hz}, 1 \mathrm{H}), 2.58 \sim 2.55(\mathrm{dd}, J=$ $16.2,3.6 \mathrm{~Hz}, 1 \mathrm{H}), 2.51 \sim 2.47(\mathrm{td}, J=11.4,3.6 \mathrm{~Hz}, 1 \mathrm{H})$, $2.46 \sim 2.20(\mathrm{~m}, 1 \mathrm{H}), 1.86(\mathrm{~s}, 3 \mathrm{H}), 1.82(\mathrm{~s}, 3 \mathrm{H}) ;{ }^{13} \mathrm{C} \mathrm{NMR}$ $\left(\mathrm{CDCl}_{3}, 150 \mathrm{MHz}\right) \delta: 174.1,173.9,147.1,146.7,134.7$, $134.5,131.1,131.0,126.6,126.4,123.5,122.6,122.4$,
$122.3,115.7,115.6,115.5,115.1,113.0,111.4,106.5$, $106.4,51.6,51.3,50.0,46.2,38.6,35.8,33.3,33.2,32.8$, $30.8,29.7,28.2,23.1,19.5$. HRMS calcd for $\mathrm{C}_{18} \mathrm{H}_{22} \mathrm{NO}_{2}$ $[\mathrm{M}+\mathrm{H}]^{+}$284.1645, found 284.1650).

辅助材料(Supporting Information) 化合物 10、11、15、 $19 、 21 、 22 、 23 、 24 、 26 、 27 a 、 27 b 、 28 、 29$ 的 ${ }^{1} \mathrm{H}$ NMR 谱图和 ${ }^{13} \mathrm{C} N \mathrm{NMR}$ 谱图, 以及化合物 $27 \mathbf{a}$ 和 $27 \mathbf{b}$ 的单晶衍 射数据. 化合物 $27 \mathrm{a}$ 和 $27 \mathrm{~b}$ 的晶体数据存于英国剑桥数 据中心，化合物 27a 的 CCDC 号为 2036649, 化合物 27b 的 CDCC 号为 2036650. 这些材料可以免费从本刊网站 (http://sioc-journal.cn/)上下载.

\section{References}

[1] Li, Q.; Jiang, J.; Fan, A.; Cui, Y.; Jia, Y. Org. Lett. 2011, 13, 312.

[2] Qin, H.; Xu, Z.; Cui, Y.; Jia, Y. Angew. Chem. Int. Ed. 2011, 50, 4447.

[3] Hu, W.; Qin, H.; Cui, Y.; Jia, Y. Chem.-Eur. J. 2013, 19, 3139.

[4] Liu, Q.; Li, Q.; Ma, Y.; Jia, Y. Org. Lett. 2013, 15, 4528.

[5] Huang, B.; Guo, L.; Jia, Y. Angew. Chem. Int. Ed. 2015, 54, 13599.

[6] Li, L.; Yuan, K.; Jia, Q.; Jia, Y. Angew. Chem. Int. Ed. 2019, 58,6074 .

[7] Liu, H.; Chen, L.; Yuan, K.; Jia, Y. Angew. Chem. Int. Ed. 2019, 58, 6362.

[8] Zhang, X.; Cai, X.; Huang, B.; Guo, L.; Gao, Z.; Jia, Y. Angew. Chem. Int. Ed. 2019, 58, 13380.

[9] Shi, S.; Yuan, K.; Jia, Y. Chin. Chem. Lett. 2020, 31, 401.

[10] Shan, D.; Jia, Y. Chin. J. Org. Chem. 2013, 33, 1144.

[11] Liu, H.; Jia, Y. Nat. Prod. Rep. 2017, 34, 411.

[12] Yuan, K.; Jia, Y. Chin. J. Org. Chem. 2018, 38, 2386.

[13] (a) Chang, P. K.; Ehrlich, K. C.; Fujii, I. Toxins 2009, 1, 74. (b) Liu, X.; Walsh, C. T. Biochemistry 2009, 48, 8746

[14] Kropp, P. J.; Breton, G. W.; Craig, S. L.; Crawford, S. D.; Durland, W. F.; Jones, J. E.; Raleigh, J. S. J. Org. Chem. 1995, 60, 4146.

[15] Tietze, L.; Beifuss, U. Synthesis 1988, 359.

[16] Snider, B. B.; Rodini, D. J.; van Straten, J. J. Am. Chem. Soc. 1980, $102,5872$.

[17] Lau, S. Y. W. Org. Lett. 2011, 13, 347.

[18] Lu, D.; Wan, Y.; Kong, L.; Zhu, G. Org. Lett. 2017, 19, 2929.

[19] Wang, R.; Chen, Y.; Shu, M.; Zhao, W.; Tao, M.; Du, C.; Fu, X.; Li, A.; Lin, Z. Chem.-Eur. J. 2020, 26, 1941.

[20] Sabitha, G.; Shankaraiah, K.; Sindhu, K.; Latha, B. Synthesis 2015, $47,124$.

[21] Liu, X.; Liu, S.; Wang, Q.; Zhou, G.; Yao, L.; Ouyang, Q.; Jiang, R.; Lan, Y.; Chen, W. Org. Lett. 2020, 22, 3149.

[22] Yu, T. Y.; Wei, H.; Luo, Y. C.; Wang, Y.; Wang, Z. Y.; Xu, P. F. J. Org. Chem. 2016, 81, 2730.

[23] Thirupathi, B.; Breitler, S.; Mahender Reddy, K.; Corey, E. J. J. Am. Chem. Soc. 2016, 138, 10842.

[24] Chou, T. H.; Yu, B. H.; Chein, R. J. Chem. Commun. 2019, 55, 13522.

[25] Ferreira, P. M. T.; Monteiro, L. S.; Pereira, G.; Ribeiro, L.; Sacramento, J.; Silva, L. Eur. J. Org. Chem. 2007, 35, 5934. 